\title{
A Review of Dynamic Vehicle Routing Problems
}

\author{
Victor Pillac $^{1,2}$, Michel Gendreau ${ }^{3,4}$, Christelle GuÉRET ${ }^{*, 1}$, \\ and Andrés L. MedaGlia ${ }^{2}$ \\ ${ }^{1}$ École des Mines de Nantes, IRCCyN, UMR CNRS 6597, Nantes, France \\ ${ }^{2}$ Universidad de los Andes, Industrial Engineering Department, Bogotá, Colombia \\ ${ }^{3}$ École Polytechnique de Montréal, Département de Mathématiques et de Génie \\ Industriel, Montréal, Canada \\ ${ }^{4}$ Centre Interuniversitaire de Recherche sur les Reseaux d'Entreprise, la \\ Logistique et le Transport (CIRRELT)
}

September 2012

The present work has been accepted for publication in European Journal of Operational Research doi:10.1016/j.ejor.2012.08.015

The orginal publication is available at elsevier.com

\begin{abstract}
A number of technological advances have led to a renewed interest on dynamic vehicle routing problems. This survey classifies routing problems from the perspective of information quality and evolution. After presenting a general description of dynamic routing, we introduce the notion of degree of dynamism, and present a comprehensive review of applications and solution methods for dynamic vehicle routing problems.
\end{abstract}

\footnotetext{
*Corresponding author: gueret@mines-nantes.fr
} 


\section{Introduction}

The Vehicle Routing Problem (VRP) formulation was first introduced by Dantzig and Ramser [35], as a generalization of the Traveling Salesman Problem (TSP) presented by Flood [49]. The VRP is generally defined on a graph $G=(\mathcal{V}, \mathcal{E}, C)$, where $\mathcal{V}=\left\{v_{0}, \ldots, v_{n}\right\}$ is the set of vertices; $\mathcal{E}=\left\{\left(v_{i}, v_{j}\right) \mid\left(v_{i}, v_{j}\right) \in \mathcal{V}^{2}, i \neq j\right\}$ the arc set; and $C=\left(c_{i j}\right)_{\left(v_{i}, v_{j}\right) \in \mathcal{E}}$ a cost matrix defined over $\mathcal{E}$, representing distances, travel times, or travel costs. Traditionally, vertex $v_{0}$ is called the depot, while the remaining vertices in $\mathcal{V}$ represent customers (or requests) that need to be serviced. The VRP consists in finding a set of routes for $K$ identical vehicles based at the depot, such that each of the vertices is visited exactly once, while minimizing the overall routing cost.

Beyond this classical formulation, a number of variants have been studied. Among the most common are the Capacitated VRP (CVRP), where each customer has a demand for a good and vehicles have finite capacity; the VRP with Time Windows (VRPTW), where each customer must be visited during a specific time frame; the VRP with Pick-up and Delivery (PDP), where goods have to be picked-up and delivered in specific amounts at the vertices; and the Heterogeneous fleet VRP (HVRP), where vehicles have different capacities. Routing problems that involve moving people between locations are referred to as Dial-A-Ride-Problem (DARP) for land transport; or Dial-A-Flight-Problem (DAFP), for air transport.

In contrast to the classical definition of the vehicle routing problem, realworld applications often include two important dimensions: evolution and quality of information [116]. Evolution of information relates to the fact that in some problems the information available to the planner may change during the execution of the routes, for example, with the arrival of new customer requests. Quality of information reflects possible uncertainty on the available data, for instance, when the demand of a customer is only known as a range estimate of its real demand. In addition, depending on the problem and the available technology, vehicle routes can either be designed statically (apriori) or dynamically. For instance, the VRP with Stochastic Demands (VRPSD), can be seen from both perspectives. From a static perspective, the problem is to design a set of robust routes a-priori, that will undergo minor changes during their execution [16, 53]. From a dynamic perspective, the problem consists in designing the vehicle routes in an online fashion, communicating to the vehicle which customer to serve next as soon as it 
becomes idle [104, 127, 128]. Based on these dimensions, Table 1 identifies four categories of routing problems.

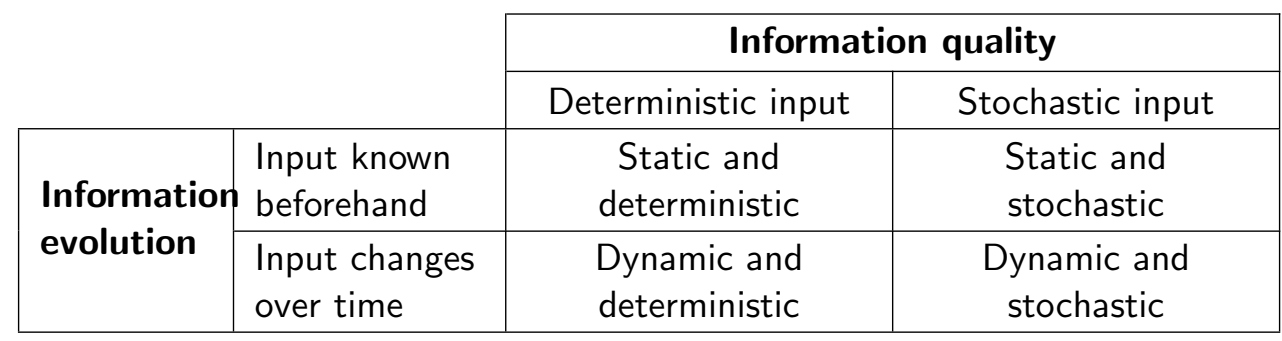

Table 1: Taxonomy of vehicle routing problems by information evolution and quality.

In static and deterministic problems, all input is known beforehand and vehicle routes do not change once they are in execution. This classical problem has been extensively studied in the literature, and we refer the interested reader to the recent reviews of exact and approximate methods by Baldacci et al. 44, Cordeau et al. [33], Laporte [83, 84, and Toth and Vigo [143].

Static and stochastic problems are characterized by input partially known as random variables, which realizations are only revealed during the execution of the routes. Additionally, it is assumed that routes are designed apriori and only minor changes are allowed afterwards. For instance, allowable changes include planning a trip back to the depot or skipping a customer. Applications in this category do not require any technological support. Uncertainty may affect any of the input data, yet the three most studied cases are 33]: stochastic customers, where a customer needs to be serviced with a given probability [15, 147]; stochastic times, in which either service or travel times are modeled by random variables [79, 85, 146]; and lastly, stochastic demands [31, 37, 86, 97, 98, 126, 128]. Further details on the static stochastic vehicle routing can be found in the reviews by Bertsimas and Simchi-Levi [16], Cordeau et al. [33], and Gendreau et al. [53].

In dynamic and deterministic problems, part or all of the input is unknown and revealed dynamically during the design or execution of the routes. For these problems, vehicle routes are redefined in an ongoing fashion, requiring technological support for real-time communication between the vehicles and the decision maker (e.g., mobile phones and global positioning systems). This class of problems are also referred to as online or real time by some 
authors [77].

Similarly, dynamic and stochastic problems have part or all of their input unknown and revealed dynamically during the execution of the routes, but in contrast with the latter category, exploitable stochastic knowledge is available on the dynamically revealed information. As before, the vehicle routes can be redefined in an ongoing fashion with the help of technological support.

Besides dynamic routing problems, where customer visits must be explicitly sequenced along the routes, there are other related vehicle dispatching problems, such as managing a fleet of emergency vehicles [23, 54, 66], or the so-called dynamic allocation problems in the area of long haul truckload trucking [60, 109, 134]. In this paper, we focus solely on dynamic problems with an explicit routing dimension.

The remainder of this document is organized as follows. Section 2 presents a general description of dynamic routing problems and introduce the notion of degree of dynamism. Section 3 reviews different applications in which dynamic routing problems arise, while Section 4 provides a comprehensive survey of solution approaches. Finally, Section 5 concludes this paper and gives directions for further research.

\section{Dynamic vehicle routing problems}

\subsection{A general definition}

The first reference to a dynamic vehicle routing problem is due to Wilson and Colvin [148]. They studied a single vehicle DARP, in which customer requests are trips from an origin to a destination that appear dynamically. Their approach uses insertion heuristics able to perform well with low computational effort. Later, Psaraftis [116] introduced the concept of immediate request: a customer requesting service always wants to be serviced as early as possible, requiring immediate replanning of the current vehicle route.

A number of technological advances have led to the multiplication of real-time routing applications. With the introduction of the Global Positioning System (GPS) in 1996, the development and widespread use of mobile and smart phones, combined with accurate Geographic Information Systems (GIS), companies are now able to track and manage their fleet in real time and cost effectively. While traditionally a two-step process (i.e., plan-execute), vehicle routing can now be done dynamically, introducing greater oppor- 
tunities to reduce operational costs, improve customer service, and reduce environmental impact.

The most common source of dynamism in vehicle routing is the online arrival of customer requests during the operation. More specifically, requests can be a demand for goods [2, 62, 70, 71, 75, 99, 101, 144] or services [7, 11, 17, 52, 89, 141. Travel time, a dynamic component of most real-world applications, has been recently taken into account [1, 6, 28, 48, 64, 65, 94, 108, 136, 139, 153; while service time has not been explicitly studied (but can be added to travel time). Finally, some recent work considers dynamically revealed demands for a set of known customers [104, 105, 126, 128] and vehicle availability [92, 93, 103], in which case the source of dynamism is the possible breakdown of vehicles. In the following we use the prefix "D-" to label problems in which new requests appear dynamically.

To better understand what we mean by dynamic, Figure 1 illustrates the route execution of a single vehicle D-VRP. Before the vehicle leaves the depot (time $t_{0}$ ), an initial route plans to visit the currently known requests $(A, B, C, D, E)$. While the vehicle executes its route, two new requests $(X$ and $Y$ ) appear at time $t_{1}$ and the initial route is adjusted to fulfill them. Finally, at time $t_{f}$ the executed route is $(A, B, C, D, Y, E, X)$.

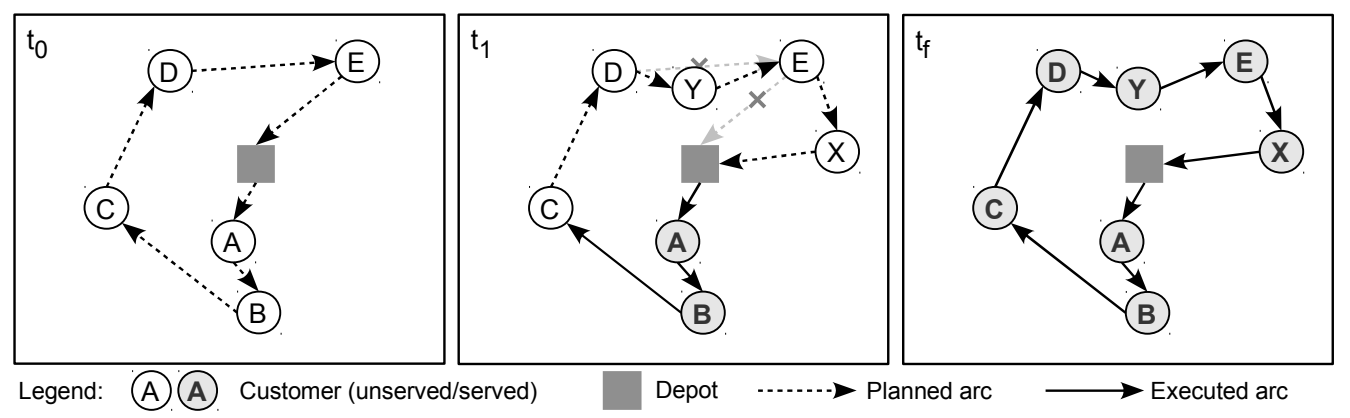

Figure 1: Example of dynamic vehicle routing

This example reveals how dynamic routing inherently adjusts routes in an ongoing fashion, which requires real-time communication between vehicles and the dispatching center. Figure 2 illustrates this real-time communication scheme, where the environment refers to the real-world while the dispatcher is the agent that gives instructions to the vehicle. Once the vehicle is ready (first dotted arrow), the dispatcher makes a decision and instructs the vehicle 
to fulfill request $A$ (first double-headed arrow). When the vehicle starts (second dotted arrow) and ends (third dotted arrow) service at request $A$, it notifies the dispatcher, which in turns updates the available information and communicates the vehicle its next request (second double-headed arrow).

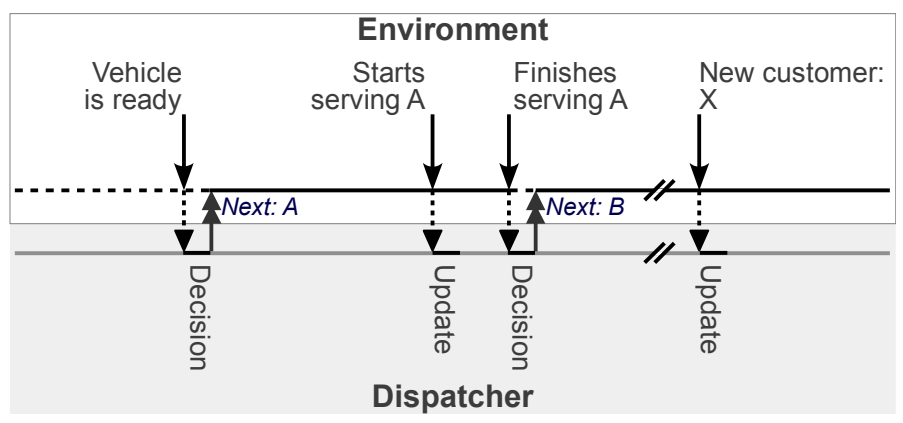

Figure 2: Timeline of events for the dynamic routing of a single vehicle

\subsection{Differences with static routing}

In contrast to their static counterparts, dynamic routing problems involve new elements that increase the complexity of their decisions (more degrees of freedom) and introduce new challenges while judging the merit of a given route plan.

In some contexts, such as the pick-up of express courier [52], the transport company may deny a customer request. As a consequence, it can reject a request either because it is simply impossible to service it, or because the cost of serving it is too high. This process of acceptance/denial has been used in many approaches [2, 43, 52, 73, 75, 92] and is referred to as service guarantee [145].

In dynamic routing, the ability to redirect a moving vehicle to a new request nearby allows for additional savings. Nevertheless, it requires realtime knowledge of the vehicle position and being able to communicate quickly with drivers to assign them new destinations. Thus, this strategy has received limited interest, with the main contributions being the early work by Regan et al. [120, 121, 122], the study of diversion issues by Ichoua et al. [73], and the work by Branchini et al. [21].

Dynamic routing also frequently differs in the objective function [118. In particular, while a common objective in the static context is the minimiza- 
tion of the routing cost, dynamic routing may introduce other notions such as service level, throughput (number of serviced requests), or revenue maximization. Having to answer to dynamic customer requests also introduces the notion of response time: a customer might request to be serviced as soon as possible, in which case the main objective may become to minimize the delay between the arrival of a request and its service.

Dynamic routing problems require making decisions in an online manner, which often compromises reactiveness with decision quality. In other words, the time invested searching for better decisions, comes at the price of a lower reactiveness to input changes. This aspect is of particular importance in contexts where customers call for a service and a good decision must be made as fast as possible.

\subsection{Measuring dynamism}

Different problems (or instances of a same problem) can have different levels of dynamism, which can be characterized according to two dimensions [76]: the frequency of changes and the urgency of requests. The former is the rate at which new information becomes available, while the latter is the time gap between the disclosure of a new request and its expected service time. From this observation three metrics have been proposed to measure the dynamism of a problem (or instance).

Lund et al. [95] defined the degree of dynamism $\delta$ as the ratio between the number of dynamic requests $n_{d}$ and the total number of requests $n_{t o t}$ as follows:

$$
\delta=\frac{n_{d}}{n_{t o t}}
$$

Based on the fact that the disclosure time of requests is also important [117, 118, Larsen [87] proposed the effective degree of dynamism $\delta^{e}$. This metric can be interpreted as the normalized average of the disclosure times. Let $T$ be the length of the planning horizon, $\mathcal{R}$ the set of requests, and $t_{i}$ the disclosure time of request $i \in \mathcal{R}$. Assuming that requests known beforehand have a disclosure time equal to $0, \delta^{e}$ can be expressed as:

$$
\delta^{e}=\frac{1}{n_{\text {tot }}} \sum_{i \in \mathcal{R}} \frac{t_{i}}{T}
$$

Larsen [87] also extended the effective degree of dynamism to problems with time windows to reflect the level of urgency of requests. He defines the 
reaction time as the difference between the disclosure time $t_{i}$ and the end of the corresponding time window $l_{i}$, highlighting that longer reaction times mean more flexibility to insert the request into the current routes. Thus, the effective degree of dynamism measure is extended as follows:

$$
\delta_{T W}^{e}=\frac{1}{n_{t o t}} \sum_{i \in \mathcal{R}}\left(1-\frac{l_{i}-t_{i}}{T}\right)
$$

It is worth noting that these three metrics only take values in the interval $[0,1]$ and all increase with the level of dynamism of a problem. Larsen et al. [88, 90] use the effective degree of dynamism to define a framework classifying D-VRPs among weakly, moderately, and strongly dynamic problems, with values of $\delta^{e}$ being respectively lower than 0.3 , comprised between 0.3 and 0.8 , and higher than 0.8 .

Although the effective degree of dynamism and its variations have proven to capture well the time-related aspects of dynamism, it could be argued that they do not take into account other possible sources of dynamism. In particular, the geographical distribution of requests, or the traveling times between requests, are also of great importance in applications aiming at the minimization of response time. Although not considered, the frequency of updates in problem information has a dramatical impact on the time available for optimization.

\section{A review of applications}

Recent advances in technology have allowed the emergence of a wide new range of applications for vehicle routing. In particular, the last decade has seen the development of Intelligent Transport Systems (ITS), which are based on a combination of geolocation technologies, with precise geographic information systems, and increasingly efficient hardware and software for data processing and operations planning. We refer the interested reader to the study by Crainic et al. [34] for more details on ITS and the contributions of operations research to this relatively new domain.

Among the ITS, the Advanced Fleet Management Systems (AFMS) are specifically designed for managing a corporate vehicle fleet. The core problem is generally to deliver (pick-up) goods or persons to (from) locations distributed in a given area. While customer requests can either be known in advance or appear dynamically during the day, vehicles are dispatched 
and routed in real time, potentially, by taking into account changing traffic conditions, uncertain demands, or varying service times. A key technological feature of AFMS is the optimization component. Traditionally, vehicle routing relies on teams of human dispatchers, meaning a critical operational process is bound to the competence and experience of dispatchers, as well as the management costs that are directly linked to the size of the fleet [1]. Advances in computer science have allowed a technological transfer from operational research to AFMS, as presented in the studies by Attanasio et al. [1], Du et al. [38], Godfrey and Powell [60], Powell and Topaloglu [115], Roy [125], Simao et al. [129], and Slater [130].

The remainder of this section presents applications where dynamic routing has been or can be implemented. The interested reader is also referred to the work by Gendreau and Potvin [55] and Ichoua et al. [76] for complementary reviews.

\subsection{Services}

In this category of applications, a service request is defined by a customer location and a possible time window; while vehicle routes just fulfill service requests without considering side constraints such as capacity. Perhaps the simplest, yet most illustrative case in this category is the dynamic traveling salesman problem 89.

A common application of dynamic routing can be found in the area of maintenance operations. Maintenance companies are often committed by contract to their customers, which specify periodical or planned visits to perform preventive maintenance, and may also request corrective maintenance on short notice. Therefore, each technician is first given a route with known requests at the beginning of the day, while new urgent requests are inserted dynamically throughout the day. An interesting feature of this problem is the possible mix of skills, tools, and spare part requirements, which have to be matched in order to service the request. This problem has been studied by Borenstein et al. [19] with an application to British Telecom.

Another application of dynamic routing arises in the context of the French non-profit organization SOS Médecins. This organization operates with a crew of physicians, who are called on duty via a call center coordinated with other emergency services. When a patient calls, the severity of the case is evaluated, and a visit by a practitioner is planned accordingly. As in other emergency services, having an efficient dispatching system reduces the 
response time, thus improving service level for the society. On the other hand, it is important to decide in real-time whether or not to send a physician, so that it is possible to ensure a proper service level in areas where emergencies are likely to appear.

Dynamic aspects can also appear on arc routing problems. This is for instance the case in the study by Tagmouti et al. [136] on the operation of a fleet of vehicles for winter gritting applications. Their work consider a network of streets or road segments that need to be gritted when affected by a moving storm. Depending on the movements of the storm, new segments may have to be gritted, and the routing of vehicles has to be updated accordingly.

\subsection{Transport of goods}

Due to the fact that urban areas are often characterized by highly variable traveling times, transport of goods in such areas have led to the definition of a specific category of applications known as city logistics. City logistics can be defined as an integrated vision of transport activities in urban areas, taking into account factors such as traffic and competition or cooperation between transport companies [140]. Barcelo et al. [6] developed a general framework for city logistics applications. They describe the different modules ranging from modeling the city road network and acquiring real-time traffic data to the dynamic routing of a fleet of vehicles. Zeimpekis et al. [153. proposed a Decision Support System (DSS) for city logistics which takes into account dynamic travel and service times.

A typical application in city logistics is the courier service present in most urban areas. Couriers are dispatched to customer locations to collect packages, and either deliver them to their destination (short haul) or to a unique depot (long haul). Depending on the level of service paid by the customer, couriers may consolidate pick-ups from various customers, or provide an expedited service. Companies offering courier services often have an heterogeneous fleet composed of bicycles, motorbikes, cars, and small vans. The problem is then to dynamically route couriers, taking into account not only the known requests, their type, pick-up and delivery locations, and time windows, but also considering traffic conditions and varying travel times. A case study by Attanasio et al. [1] outlines the benefits of using an optimizationenabled AFMS at eCourier Ltd, a London based company offering courier services. The authors illustrate that aside from the improvements in service quality, response time, and courier efficiency, the use of an automated system 
allows decoupling the fleet size from the need for more dispatchers. Further results motivated by a similar application can be found in Gendreau et al. [51] and Ghiani et al. [59].

The delivery of newspapers and magazines is a domain in which customer satisfaction is of first importance. When a magazine or newspaper is not delivered, a subscriber contacts a call center and is offered to choose between a voucher or a future delivery. In the latter case, the request is then forwarded to the delivery company, which assigns it to a driver that will do a priority delivery. Traditionally, this process relies on an exchange of phone calls, faxes, and printed documents, that ultimately communicate the driver about the pending delivery, once he/she comes back to the depot. As an alternative, Bieding et al. [18] propose a centralized application that makes use of mobile phones to communicate with drivers and intelligently perform the routing in real time, reducing costs and improving customer satisfaction. More recently, Ferrucci et al. 44] developed an approach that makes use historical data to anticipate future requests.

Another application in which customer requests need to be answered with short delays can be found in companies with a direct service model, such as grocery delivery services. In general, the customer selects products on a website, and then chooses a time frame for the delivery at his home. Traditionally, the vendor defines an arbitrary number of customers that can be serviced within a time window, and the time window is made unavailable to customers as soon as the capacity is reached. Campbell and Savelsbergh [24] defined the Home Delivery Problem, in which the goal is to maximize the total expected revenue by dynamically deciding whether or not to accept a customer request within a specific time window. In comparison with the traditional approach, this means that the time windows available for a customer are dynamically defined taking into consideration the possible future requests. The authors propose a Greedy Randomized Adaptive Search Procedure (GRASP) and compare different cost functions to capture the problem uncertainty. Later, Azi et al. 3] proposed an Adaptive Large Neighborhood Search (ALNS) that take into account uncertainty by generating scenarios containing possible demand realizations.

Apart from classical routing problems, related operational problems also arise in many organizations. The review by Stahlbock and Voss [135] on operations research applications in container terminals describes the dynamic stacker crane problem [5, 14], which considers the routing of container carriers loading and unloading ships in a terminal. Other applications include 
transport of goods inside warehouses [132, factories, and hospitals, where documents or expensive medical instruments must be transferred efficiently between services [45].

\subsection{Transport of persons}

The transport of persons is in general-and by many aspects-similar to the transport of goods, yet it is characterized by additional constraints such as regulation on waiting, travel, and service times.

Taxis are arguably the most common on-demand individual transport systems. Requests are composed of a pick-up location and time, possibly coupled with a destination. They can be either known in advance, for instance when a customer books a cab for the next day, or they can arrive dynamically, in which case a taxi must be dispatched in the shortest time. When customers cannot share a vehicle, the closest free taxi is generally the one which takes the ride, leaving limited space for optimization. The study by Caramia et al. [25], generalized by Fabri and Recht [42], focuses on a multi-cab metropolitan transportation system, where a taxi can transport more than one passenger at the same time. In this case the online algorithms minimize the total traveled distance, while assigning requests to vehicles and computing the taxi routes. This multi-cab transportation system can be generalized as an on-demand or door-to-door transport service.

Many applications involve the transport of children, the elderly, disabled people, or patients, from their home to schools, place of work, or medical centers. Xiang et al. [149] studied a DARP with changing travel speeds, vehicle breakdowns, and traffic congestion; while Dial [36], followed by Horn [67, 68, 69, studied demand-responsive transport systems. An extensive review of this class of problems can be found in the studies by Cordeau et al. [32] and Berbeglia et al. [14.

A singular application of on-demand transportation systems can be found in major hospitals, with services possibly spread across various buildings on several branches. Depending on the medical procedure or facility capacity, a patient may need to be transferred on short notice from one service to another, possibly requiring trained staff or specific equipment for his/her care. This application has been studied by Beaudry et al. [7], Kergosien et al. [80], and Melachrinoudis et al. [96].

Air taxis developed as a flexible response to the limitations of traditional airlines. Air taxis offer passengers the opportunity to travel through smaller 
airports, avoiding waiting lines at check-in and security checks. Air taxi companies offer an on-demand service: customers book a flight a few days in advance, specifying whether they are willing to share the aircraft, stop at an intermediate airport, or have flexible traveling hours. Then, the company accommodates these requests, trying to consolidate flights whenever possible. The underlying optimization problems have not been subject to much attention, except in the studies by Cordeau et al. [32, Espinoza et al. [40, 41, Fagerholt et al. [43, and Yao et al. [152. Similar problems arises in helicopter transportation systems, typically used by oil and gas companies to transport personnel between offshore petroleum platforms [63, 124].

\section{Solution Methods}

Few research was conducted on dynamic routing between the work of Psaraftis [116] in 1980 and the late 1990s. However, the last decade has seen a renewed interest for this class of problems [39], with solution techniques ranging from linear programming to metaheuristics. This section presents the major contributions in this field, and the reader is referred to the reviews, books, and special issues by Gendreau and Potvin [55, 56], Ghiani et al. [57], Goel [61, Ichoua [72], Ichoua et al. [75, 76], Jaillet and Wagner [78], Larsen et al. [91], and Zeimpekis et al. [154], to complement our review.

\subsection{Dynamic and deterministic routing problems}

This section presents approaches that have been successfully applied to dynamic routing, in the absence of stochastic information. In this context, critical information is revealed over time, meaning that the complete instance is only known at the end of the planning horizon. As a consequence, exact methods only provide an optimal solution for the current state, but do not guarantee that the solution will remain optimal once new data becomes available. Therefore, most dynamic approaches rely on heuristics that quickly compute a solution to the current state of the problem. Approaches

for dynamic and deterministic vehicle routing problems can be divided into two categories: those based on periodic reoptimization, and those based on continuous reoptimization. 


\subsubsection{Periodic reoptimization}

To the best of our knowledge, the first periodic reoptimization approach is due to Psaraftis [116], with the development of a dynamic programming approach. His research focuses on the DARP and consists in finding the optimal route each time a new request is known. The main drawback of dynamic programming is the well-known curse of dimensionality [110, Chap. 1], which prevents its application to large instances.

More generally, periodic reoptimization approaches start at the beginning of the day with a first optimization that produces an initial set of routes. Then, an optimization procedure periodically solves a static problem corresponding to the current state, either whenever the available data changes, or at fixed intervals of time -referred to as decision epochs [29] or time slices [81. The advantage of periodic reoptimization is that it can be based on algorithms developed for static routing, for which extensive research has been carried out. The main drawback is that all the optimization needs to be performed before updating the routing plan, thus increasing delays for the dispatcher.

Yang et al. [150] addressed the real-time truckload PDP, in which a fleet of trucks has to service point-to-point transport requests arriving dynamically. Important assumptions are that all trucks can only handle one request at a time, with no possible preemption, and they travel at the same constant speed. The authors propose MYOPT, a rolling horizon approach based on a linear program (LP) that is solved whenever a new request arrives. Along the same line of linear programming, Chen and Xu [29] designed a dynamic column generation algorithm (DYCOL) for the D-VRPTW. The authors propose the concept of decision epochs over the planning horizon, which are the dates when the optimization process runs. The novelty of their approach relies on dynamically generating columns for a set-partitioning model, using columns from the previous decision epoch. The authors compared DYCOL to a traditional column generation with no time limit (COL). Computational results based on the Solomon benchmark [133] demonstrate that DYCOL yields comparable results in terms of objective function, but with running times limited to 10 seconds, opposed to the various hours consumed by COL.

Montemanni et al. [102] developed an Ant Colony System (ACS) to solve the D-VRP. Similar to Kilby et al. [81], their approach uses time slices, that is, they divide the day in periods of equal duration. A request arriving during a time slice is not handled until the end of the time bucket, thus the problem 
solved during a time slice only considers the requests known at its beginning. Hence, the optimization is run statically and independently during each time slice. The main advantage of this time partition is that similar computational effort is allowed for each time slice. This discretization is also possible by the nature of the requests, which are never urgent, and can be postponed. An interesting feature of their approach is the use of the pheromone trace to transfer characteristics of a good solution to the next time slice. A similar approach was also used by Gambardella et al. [50] and Rizzoli et al. [123.

\subsubsection{Continuous reoptimization}

Continuous reoptimization approaches perform the optimization throughout the day and maintain information on good solutions in an adaptive memory [138. Whenever the available data changes, a decision procedure aggregates the information from the memory to update the current routing. The advantage is that the computational capacity is maximized, possibly at the expense of a more complex implementation. It is worth noting that because the current routing is subject to change at any time, vehicles do not know their next destination until they finish the service of a request.

To the best of our knowledge, the first continuous reoptimization approach is due Gendreau et al. [52] with the adaptation of the parallel Tabu Search (TS) framework introduced by Taillard et al. [137] to a D-VRPTW problem arising in the local operation of long distance express courier services. Their approach maintains a pool of good routes-the adaptive memorywhich is used to generate initial solutions for a parallel TS. The parallelized search is done by partitioning the routes of the current solution, and optimizing them in independent threads. Whenever a new customer request arrives, it is checked against all the solutions from the adaptive memory to decide whether it should be accepted or rejected. This framework was also implemented for the D-VRP [73, 74], while other variations of TS have been applied to the D-PDP [6, 27] and the DARP [2, 7].

Bent and Van Hentenryck [9] introduced the Multiple Plan Approach (MPA) as a generalization of the TS with adaptive memory [52]. The general idea is to populate and maintain a solution pool (the routing plans) that are used to generate a distinguished solution. Whenever a new request arrives, a procedure is called to check whether it can be serviced or not; if it can be serviced, then the request is inserted in the solution pool and incompatible solutions are discarded. Pool updates are performed periodically or whenever 
a vehicle finishes servicing a customer. This pool-update phase is crucial and ensures that all solutions are coherent with the current state of vehicles and customers. The pool can be seen as an adaptive memory that maintains a set of alternative solutions.

In an early work, Benyahia and Potvin [13] studied the D-PDP and proposed a Genetic Algorithm (GA) that models the decision process of a human dispatcher. More recently, GAs were also used for the same problem [30, 65] and for the D-VRP [144]. Genetic algorithms in dynamic contexts are very similar to those designed for static problems, although they generally run throughout the planning horizon and solutions are constantly adapting to the changes made to the input.

\subsection{Dynamic and stochastic routing problems}

Dynamic and stochastic routing problems can be seen as an extension of their deterministic counterparts, where additional (stochastic) knowledge is available in the dynamically revealed input. Approaches for this class of problems can be divided in two categories: those based on sampling and those based on stochastic modeling. As their name suggests, sampling strategies incorporate stochastic knowledge by generating scenarios based on realizations drawn from random variable distributions. Each scenario is then optimized by solving the static and deterministic problem they define. On the other hand, approaches based on stochastic modeling integrate stochastic knowledge analytically. The advantage of sampling is its relative simplicity and

flexibility on distributional assumptions, while its drawback is the massive generation of scenarios to accurately reflect reality. Alternatively, stochastic modeling strategies formally capture the stochastic nature of the problem, but they are highly technical in their formulation and require to efficiently compute possibly complex expected values. Examples of these two strategies follow.

\subsubsection{Stochastic modeling}

Powell et al. [113] formulated a truckload PDP as a Markov Decision Process (MDP). Later, MDPs were used by Thomas and White [142] and Thomas [141] to solve a VRP in which known customers may ask for service with a known probability. Kim et al. 82. also used MDPs to tackle the VRP with dynamic travel times. Unfortunately, the curse of dimensionality and the 
simplifying assumptions make this approach unsuitable in most real-world applications. Nonetheless, it allowed new insights in the field of dynamic programming.

To cope with the scalability problems of traditional dynamic programming, Approximate Dynamic Programming (ADP) steps forward in time, approximates the value function, and ultimately avoids the evaluation of all possible states. We refer the interested reader to Powell [110, 111] for a more detailed description of the ADP framework. ADP has been successfully applied to freight transport [112, 114] and fleet management problems [60, 115, 129]. In particular, Novoa and Storer [104] propose an ADP algorithm to dynamically solve the VRPSD.

Linear programming has also been adapted to the dynamic and stochastic context. The OPTUN approach, proposed by Yang et al. [150] as an extension of MYOPT (see $\S$ 4.1.1), considers opportunity costs on each arc to reflect the expected cost of traveling to isolated areas. Consequently, the optimization tends to reject isolated requests, and avoids traversing arcs that are far away from potential requests. Later, Yang et al. [151] studied the emergency vehicle dispatching and routing and proposed a mathematical formulation that was later used by Haghani and Yang [66] on a similar problem.

\subsubsection{Sampling}

Sampling approaches rely on the generation of scenarios containing possible realizations of the random variables. Figure 3 illustrates how scenarios are generated for the D-VRP. Solely based on the current customers, the optimal tour would be $(A, B, E, D, C)$ (3a.), which ignores two zones (gray areas) where customers are likely to appear. By sampling the customer spatial distributions, customers $X, Y$, and $Z$ are generated, and the new optimal tour is $(C, X, Y, B, A, Z, E, D)$ (3b.). Removing the sampled (potential) customers leads to the tour $(C, B, A, E, D)$ (3c.) which is suboptimal regarding a myopic cost evaluation, but leaves room to accommodate new customers at a lower cost.

The Multiple Scenario Approach (MSA) is a predictive adaptation of the MPA framework discussed in $\S 4.1 .2$. The idea behind MSA is to take advantage of the time between decisions to continuously improve the current scenario pool. During the initialization, the algorithm, generates a first set of scenarios based on the requests known beforehand. Throughout the day, 


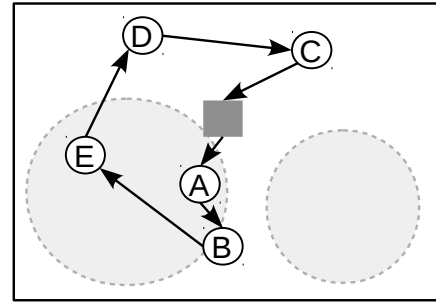

a. A-priori optimal tour

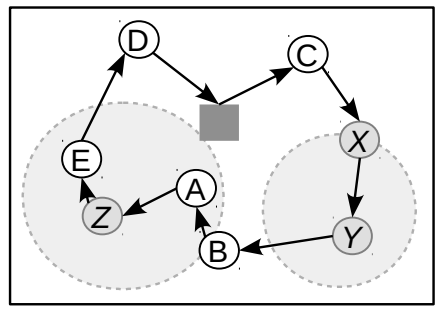

b. Optimal tour with sampled customers

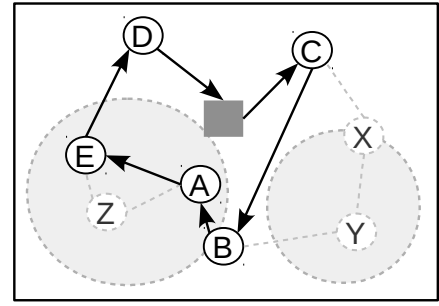

c. Optimized scenario without sampled customers

Legend: A A Customer (actual/sampled)

Depot

Customer distribution density

Figure 3: Scenario generation in sampling approaches.

scenarios are then reoptimized and new ones are generated and added to the pool. When a decision is required, the scenario optimization procedure is suspended, and MSA uses the scenario pool to select the request to service next. MSA then discards the scenarios that are incompatible with the current routing, and resumes the optimization. Computational experiments on instances adapted from the Solomon benchmark [133] showed that MSA outperforms MPA both in terms of serviced customers and traveled distances, especially for instances with high degrees of dynamism [9]. Flatberg et al. [47] adapted the SPIDER commercial solver to use multiple scenarios and a consensus algorithm to tackle the D-VRP, while Pillac et al. [107] implemented an event-driven optimization framework based on MSA and showed significant improvements over state-of-the-art algorithms for the D-VRPSD.

An important component of scenario based-approaches such as MSA is the decision process, which defines how the information from the scenario pool is used to reach upon a decision regarding the next customer to visit. The most common algorithms used to reach a decision in MSA are: consensus, expectation, and regret. The consensus algorithm [9, 10] selects the customer appearing first with the highest frequency among scenarios. Expectation [8, 10, 26] consists in evaluating the cost of visiting each customer first by forcing its visit in all scenarios and performing a complete optimization. Finally, regret [8] approximates the expectation algorithm and avoids the reoptimization of all scenarios. Even though these algorithms were initially designed for the routing of a single vehicle, they can be extended to the multi-vehicle case [145]. 
Hvattum et al. [70] developed the Dynamic Sample Scenario Hedge Heuristic (DSHH), an approach similar to the consensus algorithm for D-VRP. This method divides the planning horizon into time intervals. At the beginning of each interval, DSHH revises the routing by assigning a subset of promising requests to the vehicles, depending on the frequency of their assignment over all scenarios. DSHH later led to the development of the Branch and Regret Heuristic (BRH), where scenarios are merged to build a unique solution.

Various local search approaches have been developed for the stochastic and dynamic problems. Ghiani et al. [59] developed an algorithm for the D-PDP that only samples the near future to reduce the computational effort. The main difference with MSA is that no scenario pool is used and the selection of the distinguished solution is based on the expected penalty of accommodating requests in the near future. Azi et al. [3] developed an Adaptive Large Neighborhood Search (ALNS) for a dynamic routing problem with multiple delivery routes, in which the dynamic decision is the acceptance of a new request. The approach maintains a pool of scenarios, optimized by an ALNS, that are used to evaluate the opportunity value of an incoming request.

Tabu search has also been adapted to dynamic and stochastic problems. Ichoua et al. 75 and Attanasio et al. 1] tackled with tabu search the DVRPTW and the D-PDP, respectively.

\subsubsection{Other strategies}

In addition to the general frameworks described previously, the use of stochastic knowledge allows for the design and implementation of other strategies that try to adequately respond to upcoming events.

The waiting strategy consists in deciding whether a vehicle should wait after servicing a request, before heading toward the next customer; or planning a waiting period on a strategic location. This strategy is particularly important in problems with time windows, where time lags appear between requests. Mitrović-Minić et al. [100] proved that in all cases it is better to wait after servicing a customer, but a more refined strategy can lead to further improvements. The problem is in general to evaluate the likelihood of a new request in the neighborhood of a serviced request and to plan a waiting period accordingly. The waiting strategy has been implemented in various frameworks for the D-VRP [22, 141], D-VRPTW [12, 21, 75, 145], D-PDP [59, 100], and Dynamic and Stochastic TSP [58]. The strategy has shown 
good results, especially in the case of a limited fleet facing a high request rate $[145$.

Aside from the waiting after or before servicing a customer, a vehicle can be relocated to a strategic position, where new requests are likely to arrive. This strategy is the keystone of emergency fleet deployment, also known as Emergency Vehicle Dispatching-or Redeployment-Problem [54, 66]. The relocation strategy has also been applied to other vehicle routing problems, such as the D-VRP [87],D-VRPTW [12, 21, 75, 145], D-TSPTW [89], D-PDP [59, 119], and the Resource Allocation Problem (RAP) [60].

Request buffering, introduced by Pureza and Laporte [119], consists in delaying the assignment of some requests to vehicles in a priority buffer, so that more urgent requests can be handled first.

\subsection{Performance evaluation}

In contrast to static problems, where measuring the performance of an algorithm is straightforward (i.e., running time and solution quality), dynamic problems require the introduction of new metrics to assess the performance of a particular method.

Sleator and Tarjan [131] introduced the competitive analysis [77, 90]. Let $P$ be a minimization problem and $\mathcal{I}$ the set of all instances of $P$. Let $z^{*}\left(I_{\mathrm{off}}\right)$ be the optimal cost for the offline instance $I_{\text {off }}$ corresponding to $I \in \mathcal{I}$. For offline instance $I_{\text {off }}$, all input data from instance $I$, either static or dynamic, is available when building the solution. In contrast, the data of the online version $I$ is revealed in real time, thus an algorithm $\mathcal{A}$ has to take into account new information as it is revealed and produce a solution relevant to the current state of knowledge. Let $z_{\mathcal{A}}(I)=z\left(x_{\mathcal{A}}(I)\right)$ be the cost of the final solution $x_{\mathcal{A}}(I)$ found by the online algorithm $\mathcal{A}$ on instance $I$. Algorithm $\mathcal{A}$ is said to be $c$-competitive, or equivalently to have a competitive ratio of $c$, if there exists a constant $\alpha$ such that

$$
z_{\mathcal{A}}(I) \leq c \cdot z^{*}\left(I_{\text {off }}\right)+\alpha \quad, \quad \forall I \in \mathcal{I}
$$

In the case where $\alpha=0$, the algorithm is said to be strictly c-competitive, meaning that in all cases the objective value of the solution found by $\mathcal{A}$ will be at most of $c$ times the optimal value. The competitive ratio metric allows a worst-case absolute measure of an algorithm performance in terms of the objective value. We refer the reader to Borodin and El-Yaniv [20] for an 
in-depth analysis of this measure, and to Jaillet and Wagner [77] and Fink et al. [46] for results on various routing problems.

The main drawback of the competitive analysis is that it requires to prove the previously stated inequality analytically, which may be complex for realworld applications. The value of information proposed by Mitrović-Minić et al. 100 constitutes a more flexible and practical metric. We denote by $z_{\mathcal{A}}\left(I_{\text {off }}\right)$ the value of the objective function returned by algorithm $\mathcal{A}$ for the offline instance $I_{\text {off }}$. The value of information $V_{\mathcal{A}}(I)$ for algorithm $\mathcal{A}$ on instance $I$ is then defined as

$$
V_{\mathcal{A}}(I)=\frac{z_{\mathcal{A}}(I)-z_{\mathcal{A}}\left(I_{\text {off }}\right)}{z_{\mathcal{A}}\left(I_{\text {off }}\right)}
$$

The value of information can be interpreted as the gap between the solution returned by an algorithm $\mathcal{A}$ on a instance $I$ and the solution returned by the same algorithm when all information from $I$ is known beforehand. In contrast with the competitive ratio, the value of information gives information on the performance of an algorithm based on empirical results, without requiring optimal solutions for the offline instances. It captures the impact of the dynamism on the solution yield by the algorithm under analysis. For instance, Gendreau et al. 52] report a value of information between 2.5\% and $4.1 \%$ for their tabu search algorithm for the D-VRPTW, while Tagmouti et al. [136] reports values between $10 \%$ and $26.7 \%$ for a variable neighborhood search descent applied to a dynamic arc routing problem.

\subsection{Benchmarks}

To date, there is no reference benchmark for dynamic routing problems. Although, it is worth noting that various authors based their computational experiments on adaptations of the Solomon [133] instances for static routing [8, 9, 28, 29, 52. Van Hentenryck and Bent [145, Chap. 10] describe how the original benchmark by Solomon [133] can be adapted to dynamic problems.

The interested reader is referred to the website of Pankratz and Krypczyk [106] for an updated list of publicly available instances sets for dynamic vehicle routing problems. 


\section{Conclusions}

Recent technological advances provide companies with the right tools to manage their fleet in real time. Nonetheless, these new technologies also introduce more complexity in fleet management tasks, unveiling the need for decision support systems adapted to dynamic contexts. Consequently, during the last decade, the research community have shown a growing interest for the underlying optimization problems, leading to a new family of approaches specifically designed to efficiently address dynamism and uncertainty. By analyzing the current state of the art, some directions can be drawn for future research in this relatively new field.

First, further work should aim at creating a taxonomy of dynamic vehicle routing problem, possibly by extending existing research on static routing [39]. This would allow a more precise classification of approaches, evaluate similarities between problems, and foster the development of generic frameworks.

Second, there is currently no reference benchmark for dynamic vehicle

routing problems. Therefore, there is a strong need for the development of publicly available benchmarks for the most common dynamic vehicle routing problems.

Third, with the advent of multi-core processors on desktop computers, and low-cost graphical processing units (GPU), parallel computing is now readily available for time-consuming methods such as those based on sampling. Although early studies considered distributed optimization [52], most approaches reviewed in this document do not take advantage of parallel architectures. The development of parallel algorithms is a challenge that could reduce the time needed for optimization and provide decision makers with highly reactive tools.

Fourth, our review of the existing literature revealed that a large fraction of work done in the area of dynamic routing does not consider stochastic aspects. We are convinced that developing algorithms that make use of stochastic information will improve the fleet performance and reduce operating costs. Thus this line of research should become a priority in the near future.

Finally, researchers have mainly focused on the routing aspect of the dynamic fleet management. However, in some applications there is more that can be done to improve performance and service level. For instance, in equipment maintenance services, the call center has a certain degree of 
freedom in fixing service appointments. In other words, it means that the customer time windows can be defined, or influenced, by the call center operator. As a consequence, a system in which aside from giving a yes/no answer to a customer request, suggests convenient times for the company would be highly desirable in such contexts.

\section{Acknowledgements}

Financial support for this work was provided by the CPER (Contrat de Projet Etat Region) Vallée du Libre; and the Centro de Estudios Interdisciplinarios Básicos y Aplicados en Complejidad (CEIBA, Colombia). This support is gratefully acknowledged.

\section{References}

[1] Attanasio, A., Bregman, J., Ghiani, G., and Manni, E. (2007). Real-time fleet management at Ecourier Ltd. In Zeimpekis, V., Tarantilis, C. D., Giaglis, G. M., and Minis, I., editors, Dynamic Fleet Management, volume 38 of Operations Research/Computer Science Interfaces, chapter 10, pages 219-238. Springer US.

[2] Attanasio, A., Cordeau, J. F., Ghiani, G., and Laporte, G. (2004). Parallel tabu search heuristics for the dynamic multi-vehicle dial-a-ride problem. Parrallel Computing, 30(3):377-387.

[3] Azi, N., Gendreau, M., and Potvin, J. Y. (2011). A dynamic vehicle routing problem with multiple delivery routes. Annals of Operations Research, In-press.

[4] Baldacci, R., Toth, P., and Vigo, D. (2007). Recent advances in vehicle routing exact algorithms. 4OR: A Quarterly Journal of Operations Research, 5(4):269-298.

[5] Balev, S., Guinand, F., Lesauvage, G., and Olivier, D. (2009). Dynamical handling of straddle carriers activities on a container terminal in uncertain environment - a swarm intelligence approach -. In Proceedings of the 2009 International Conference on Complex Systems and Applications (ICCSA 2009), Le Havre, France. University of Le Havre.

[6] Barcelo, J., Grzybowska, H., and Pardo, S. (2007). Vehicle routing and scheduling models, simulation and city logistics. In Zeimpekis, V., Tarantilis, C. D., Giaglis, G. M., and Minis, I., editors, Dynamic Fleet Management, volume 38 of Operations Research/Computer Science Interfaces, pages 163-195. Springer US.

[7] Beaudry, A., Laporte, G., Melo, T., and Nickel, S. (2010). Dynamic transportation of patients in hospitals. OR Spectrum, 32:77-107. 
[8] Bent, R. and Van Hentenryck, P. (2004a). Regrets only! online stochastic optimization under time constraints. In Proceedings of the 19th National Conference on Artificial Intelligence (AAAI-04), pages 501-506. AAAI Press.

[9] Bent, R. and Van Hentenryck, P. (2004b). Scenario-based planning for partially dynamic vehicle routing with stochastic customers. Operations Research, 52(6):977-987.

[10] Bent, R. and Van Hentenryck, P. (2004c). The value of consensus in online stochastic scheduling. In Proceedings of the 14th International Conference on Automated Planning and Scheduling (ICAPS-04). AAAI Press.

[11] Bent, R. and Van Hentenryck, P. (2005). Online stochastic and robust optimization. In Maher, M., editor, Advances in Computer Science - ASIAN 2009, volume 3321 of Lecture Notes in Computer Science, pages 286-300. Springer Berlin / Heidelberg.

[12] Bent, R. and Van Hentenryck, P. (2007). Waiting and relocation strategies in online stochastic vehicle routing. In Veloso, M., editor, Proceedings of ohe 20th International Joint Conference on Artifical Intelligence (IJCAI-07), pages 1816-1821.

[13] Benyahia, I. and Potvin, J. Y. (1998). Decision support for vehicle dispatching using genetic programming. IEEE Transactions on Systems Man and Cybernetics Part A Systems and Humans, 28(3):306-314.

[14] Berbeglia, G., Cordeau, J.-F., and Laporte, G. (2010). Dynamic pickup and delivery problems. European Journal of Operational Research, 202(1):8 - 15 .

[15] Bertsimas, D. (1988). Probabilistic combinatorial optimization problems. PhD thesis, Massachusetts Institute of Technology, Dept. of Mathematics.

[16] Bertsimas, D. and Simchi-Levi, D. (1996). A new generation of vehicle routing research: robust algorithms, addressing uncertainty. Operations Research, 44(2):286-304.

[17] Bertsimas, D. and Van Ryzin, G. (1991). A stochastic and dynamic vehicle-routing problem in the Euclidean plane. Operations Research, 39(4):601-615.

[18] Bieding, T., Görtz, S., and Klose, A. (2009). On-line routing per mobile phone : A case on subsequent deliveries of newspapers. In Beckmann, M., Künzi, H. P., Fandel, G., Trockel, W., Basile, A., Drexl, A., Dawid, H., Inderfurth, K., Kürsten, W., Nunen, J. A., Speranza, M. G., and Bertazzi, L., editors, Innovations in Distribution Logistics, volume 619 of Lecture Notes in Economics and Mathematical Systems, pages 29-51. Springer Berlin Heidelberg.

[19] Borenstein, Y., Shah, N., Tsang, E., Dorne, R., Alsheddy, A., and Voudouris, C. (2010). On the partitioning of dynamic workforce scheduling problems. Journal of Scheduling, 13(4):411-425.

[20] Borodin, A. and El-Yaniv, R. (2005). Online Computation and Competitive Analysis. Cambridge University Press, Cambridge. 
[21] Branchini, R. M., Armentano, V. A., and Lokketangen, A. (2009). Adaptive granular local search heuristic for a dynamic vehicle routing problem. Computers $\&$ Operations Research, 36(11):2955-2968.

[22] Branke, J., Middendorf, M., Noeth, G., and Dessouky, M. (2005). Waiting strategies for dynamic vehicle routing. Transportation Science, 39(3):298-312.

[23] Brotcorne, L., Laporte, G., and Semet, F. (2003). Ambulance location and relocation models. European Journal of Operational Research, 147(3):451-463.

[24] Campbell, A. and Savelsbergh, M. (2005). Decision support for consumer direct grocery initiatives. Transportation Science, 39(3):313-327.

[25] Caramia, M., Italiano, G., Oriolo, G., Pacifici, A., and Perugia, A. (2002). Routing a fleet of vehicles for dynamic combined pick-up and deliveries services. In Proceedings of the Symposium on Operation Research 2001, pages 3-5, Duisburg, Germany.

[26] Chang, H., Givan, R., and Chong, E. (2000). On-line scheduling via sampling. In Proceedings of the Artificial Intelligence Planning and Scheduling (AIPS 2000), pages $62-71$.

[27] Chang, M. S., Chen, S., and Hsueh, C. (2003). Real-time vehicle routing problem with time windows and simultaneous delivery/pickup demands. Journal of the Eastern Asia Society for Transportation Studies, 5:2273-2286.

[28] Chen, H.-K., Hsueh, C.-F., and Chang, M.-S. (2006). The real-time time-dependent vehicle routing problem. Transportation Research Part E: Logistics and Transportation Review, 42(5):383-408.

[29] Chen, Z. and Xu, H. (2006). Dynamic column generation for dynamic vehicle routing with time windows. Transportation Science, 40(1):74-88.

[30] Cheung, B. K. S., Choy, K. L., Li, C.-L., Shi, W., and Tang, J. (2008). Dynamic routing model and solution methods for fleet management with mobile technologies. International Journal of Production Economics, 113(2):694-705.

[31] Christiansen, C. and Lysgaard, J. (2007). A branch-and-price algorithm for the capacitated vehicle routing problem with stochastic demands. Operations Research Letters, 35(6):773-781.

[32] Cordeau, J.-F., Laporte, G., Potvin, J.-Y., and Savelsbergh, M. W. (2007a). Transportation on demand. In Barnhart, C. and Laporte, G., editors, Transportation, volume 14 of Handbooks in Operations Research and Management Science, chapter 7, pages 429-466. Elsevier.

[33] Cordeau, J.-F., Laporte, G., Savelsbergh, M. W., and Vigo, D. (2007b). Vehicle routing. In Barnhart, C. and Laporte, G., editors, Transportation, volume 14 of Handbooks in Operations Research and Management Science, chapter 6, pages 367-428. Elsevier. 
[34] Crainic, T. G., Gendreau, M., and Potvin, J.-Y. (2009). Intelligent freighttransportation systems: Assessment and the contribution of operations research. Transportation Research Part C: Emerging Technologies, 17(6):541-557.

[35] Dantzig, G. and Ramser, J. (1959). The truck dispatching problem. Management Science, 6(1):80-91.

[36] Dial, R. B. (1995). Autonomous dial-a-ride transit introductory overview. Transportation Research Part C: Emerging Technologies, 3(5):261-275.

[37] Dror, M., Laporte, G., and Trudeau, P. (1989). Vehicle routing with stochastic demands: Properties and solution frameworks. Transportation Science, 23(3):166-176.

[38] Du, T., Wang, F. K., and Lu, P.-Y. (2007). A real-time vehicle-dispatching system for consolidating milk runs. Transportation Research Part E: Logistics and Transportation Review, 43(5):565-577.

[39] Eksioglu, B., Vural, A. V., and Reisman, A. (2009). The vehicle routing problem: A taxonomic review. Computers $\mathcal{E} 3$ Industrial Engineering, 57(4):1472 - 1483.

[40] Espinoza, D., Garcia, R., Goycoolea, M., Nemhauser, G. L., and Savelsbergh, M. W. P. (2008a). Per-seat, on-demand air transportation part I: Problem description and an integer multicommodity flow model. Transportation Science, 42(3):263-278.

[41] Espinoza, D., Garcia, R., Goycoolea, M., Nemhauser, G. L., and Savelsbergh, M. W. P. (2008b). Per-seat, on-demand air transportation part II: Parallel local search. Transportation Science, 42(3):279-291.

[42] Fabri, A. and Recht, P. (2006). On dynamic pickup and delivery vehicle routing with several time windows and waiting times. Transportation Research Part B: Methodological, 40(4):335 - 350.

[43] Fagerholt, K., Foss, B. A., and Horgen, O. J. (2009). A decision support model for establishing an air taxi service: a case study. Journal of The Operational Research Society, 60(9):1173-1182.

[44] Ferrucci, F., Block, S., and Gendreau, M. (2011). Real-time distribution of perishable goods using past request information to anticipate future requests. Operations Research, Under review:34.

[45] Fiegl, C. and Pontow, C. (2009). Online scheduling of pick-up and delivery tasks in hospitals. Journal of Biomedical Informatics, 42(4):624 - 632 .

[46] Fink, I., Krumke, S. O., and Westphal, S. (2009). New lower bounds for online k-server routing problems. Information Processing Letters, 109(11):563 - 567. 
[47] Flatberg, T., Hasle, G., Kloster, O., Nilssen, E. J., and Riise, A. (2007). Dynamic and stochastic vehicle routing in practice. In Zeimpekis, V., Tarantilis, C. D., Giaglis, G. M., and Minis, I., editors, Dynamic Fleet Management, volume 38 of Operations Research/Computer Science Interfaces, pages 41-63. Springer US.

[48] Fleischmann, B., Gnutzmann, S., and Sandvoss, E. (2004). Dynamic vehicle routing based on online traffic information. Transportation Science, 38(4):420-433.

[49] Flood, M. (1956). The traveling-salesman problem. Operations Research, 4(1):61-75.

[50] Gambardella, L., Rizzoli, A., Oliverio, F., Casagrande, N., Donati, A., Montemanni, R., and Lucibello, E. (2003). Ant colony optimization for vehicle routing in advanced logistics systems. In Proceedings of the International Workshop on Modelling and Applied Simulation (MAS 2003), pages 3-9.

[51] Gendreau, M., Guertin, F., Potvin, J.-Y., and Séguin, R. (2006). Neighborhood search heuristics for a dynamic vehicle dispatching problem with pick-ups and deliveries. Transportation Research Part C: Emerging Technologies, 14(3):157-174.

[52] Gendreau, M., Guertin, F., Potvin, J.-Y., and Taillard, E. (1999). Parallel tabu search for real-time vehicle routing and dispatching. Transportation Science, 33(4):381-390.

[53] Gendreau, M., Laporte, G., and Séguin, R. (1996). Stochastic vehicle routing. European Journal of Operational Research, 88(1):3 - 12.

[54] Gendreau, M., Laporte, G., and Semet, F. (2001). A dynamic model and parallel tabu search heuristic for real-time ambulance relocation. Parallel Computing, 27(12):1641 1653.

[55] Gendreau, M. and Potvin, J.-Y. (1998). Dynamic vehicle routing and dispatching. In Crainic, Teodor G. and Laporte, Gilbert, editors, Fleet management and logistics, chapter 5, pages 115-126. Kluwer, Boston.

[56] Gendreau, M. and Potvin, J.-Y., editors (2004). Transportation Science. Number 38. (special issue on real-time fleet management).

[57] Ghiani, G., Guerriero, F., Laporte, G., and Musmanno, R. (2003). Real-time vehicle routing: Solution concepts, algorithms and parallel computing strategies. European Journal of Operational Research, 151(1):1 - 11.

[58] Ghiani, G., Laporte, G., Manni, E., and Musmanno, R. (2008). Waiting strategies for the dynamic and stochastic traveling salesman problem. International Journal of Operations Research, 5(4):233-241.

[59] Ghiani, G., Manni, E., Quaranta, A., and Triki, C. (2009). Anticipatory algorithms for same-day courier dispatching. Transportation Research Part E: Logistics and Transportation Review, 45(1):96 - 106. 
[60] Godfrey, G. and Powell, W. B. (2002). An adaptive dynamic programming algorithm for dynamic fleet management, I: Single period travel times. Transportation Science, $36(1): 21-39$.

[61] Goel, A. (2008). Fleet Telematics: Real-time management and planning of commercial vehicle operations, volume 40 of Operations Research Computer Science Interfaces Series. Springer Verlag.

[62] Goel, A. and Gruhn, V. (2008). A general vehicle routing problem. European Journal of Operational Research, 191(3):650-660.

[63] Gribkovskaia, I., Laporte, G., and Shlopak, A. (2008). A tabu search heuristic for a routing problem arising in servicing of offshore oil and gas platforms. Journal of the Operational Research Society, 59(11):1449-1459.

[64] Güner, A. R., Murat, A., and Chinnam, R. B. (2012). Dynamic routing under recurrent and non-recurrent congestion using real-time its information. Computers $\mathscr{6}$ Operations Research, 39(2):358 - 373.

[65] Haghani, A. and Jung, S. (2005). A dynamic vehicle routing problem with timedependent travel times. Computers \&3 Operations Research, 32(11):2959 - 2986.

[66] Haghani, A. and Yang, S. (2007). Real-time emergency response fleet deployment: Concepts, systems, simulation \& case studies. In Zeimpekis, V., Tarantilis, C. D., Giaglis, G. M., and Minis, I., editors, Dynamic Fleet Management, volume 38 of Operations Research/Computer Science Interfaces, pages 133-162. Springer US.

[67] Horn, M. E. T. (2002a). Fleet scheduling and dispatching for demand-responsive passenger services. Transportation Research Part C: Emerging Technologies, 10(1):3563.

[68] Horn, M. E. T. (2002b). Multi-modal and demand-responsive passenger transport systems: a modelling framework with embedded control systems. Transportation Research Part A: Policy and Practice, 36(2):167 - 188.

[69] Horn, M. E. T. (2004). Procedures for planning multi-leg journeys with fixed-route and demand-responsive passenger transport services. Transportation Research Part C: Emerging Technologies, 12(1):33-55.

[70] Hvattum, L. M., Lokketangen, A., and Laporte, G. (2006). Solving a dynamic and stochastic vehicle routing problem with a sample scenario hedging heuristic. Transportation Science, 40(4):421-438.

[71] Hvattum, L. M., Lokketangen, A., and Laporte, G. (2007). A branch-and-regret heuristic for stochastic and dynamic vehicle routing problems. Networks, 49(4):330 340. 
[72] Ichoua, S. (2001). Problèmes de gestion de flottes de véhicules en temps réel. $\mathrm{PhD}$ thesis, Universite de Montreal, Montreal, Canada.

[73] Ichoua, S., Gendreau, M., and Potvin, J.-Y. (2000). Diversion issues in real-time vehicle dispatching. Transportation Science, 34(4):426-438.

[74] Ichoua, S., Gendreau, M., and Potvin, J.-Y. (2003). Vehicle dispatching with timedependent travel times. European Journal of Operational Research, 144(2):379 - 396.

[75] Ichoua, S., Gendreau, M., and Potvin, J.-Y. (2006). Exploiting knowledge about future demands for real-time vehicle dispatching. Transportation Science, 40(2):211225.

[76] Ichoua, S., Gendreau, M., and Potvin, J.-Y. (2007). Planned route optimization for real-time vehicle routing. In Zeimpekis, V., Tarantilis, C. D., Giaglis, G. M., and Minis, I., editors, Dynamic Fleet Management, volume 38 of Operations Research/Computer Science Interfaces, pages 1-18. Springer US.

[77] Jaillet, P. and Wagner, M. R. (2008a). Generalized online routing: New competitive ratios, resource augmentation, and asymptotic analyses. Operations Research, $56(3): 745-757$.

[78] Jaillet, P. and Wagner, M. R. (2008b). Online vehicle routing problems: A survey. In The Vehicle Routing Problem: Latest Advances and New Challenges, volume 43 of Operations Research/Computer Science Interfaces Series, pages 221-237. Springer US.

[79] Kenyon, A. S. and Morton, D. (2003). Stochastic vehicle routing with random travel times. Transportation Science, 37(1):69.

[80] Kergosien, Y., Lenté, C., Piton, D., and Billaut, J.-C. (2011). A tabu search heuristic for the dynamic transportation of patients between care units. European Journal of Operational Research, In Press, doi:10.1016/j.ejor.2011.04.033:--

[81] Kilby, P., Prosser, P., and Shaw, P. (1998). Dynamic VRPs: a study of scenarios. Technical Report APES-06-1998, University of Strathclyde, Glasgow, Scotland.

[82] Kim, S., Lewis, M., and C., W. C. (2005). Optimal vehicle routing with real-time traffic information. IEEE Transactions on Intelligent Transportation Systems, 6(2):178188.

[83] Laporte, G. (2007). What you should know about the vehicle routing problem. Naval Research Logistics, 54(8):811-819.

[84] Laporte, G. (2009). Fifty years of vehicle routing. Transportation Science, 43(4):408416.

[85] Laporte, G., Louveaux, F., and Mercure, H. (1992). The vehicle routing problem with stochastic travel times. Transportation Science, 26(3):161-170. 
[86] Laporte, G., Louveaux, F., and Van Hamme, L. (2002). An integer L-shaped algorithm for the capacitated vehicle routing problem with stochastic demands. Operations Research, 50(3):415-423.

[87] Larsen, A. (2001). The Dynamic Vehicle Routing Problem. PhD thesis, Technical University of Denmark (DTU).

[88] Larsen, A., Madsen, O. B. G., and Solomon, M. M. (2002). Partially dynamic vehicle routing - models and algorithms. The Journal of the Operational Research Society, 53(6):637-646.

[89] Larsen, A., Madsen, O. B. G., and Solomon, M. M. (2004). The a priori dynamic traveling salesman problem with time windows. Transportation Science, 38(4):459-472.

[90] Larsen, A., Madsen, O. B. G., and Solomon, M. M. (2007). Classification of dynamic vehicle routing systems. In Zeimpekis, V., Tarantilis, C. D., Giaglis, G. M., and Minis, I., editors, Dynamic Fleet Management, volume 38 of Operations Research/Computer Science Interfaces Series, chapter 2, pages 19-40. Springer US.

[91] Larsen, A., Madsen, O. B. G., and Solomon, M. M. (2008). Recent developments in dynamic vehicle routing systems. In The Vehicle Routing Problem: Latest Advances and New Challenges, volume 43 of Operations Research/Computer Science Interfaces Series, pages 199-218. Springer US.

[92] Li, J.-Q., Mirchandani, P. B., and Borenstein, D. (2009a). A lagrangian heuristic for the real-time vehicle rescheduling problem. Transportation Research Part E: Logistics and Transportation Review, 45(3):419-433.

[93] Li, J.-Q., Mirchandani, P. B., and Borenstein, D. (2009b). Real-time vehicle rerouting problems with time windows. European Journal of Operational Research, 194(3):711 727.

[94] Lorini, S., Potvin, J.-Y., and Zufferey, N. (2011). Online vehicle routing and scheduling with dynamic travel times. Computers \&3 Operations Research, 38(7):1086 - 1090.

[95] Lund, K., Madsen, O. B. G., and Rygaard, J. M. (1996). Vehicle routing problems with varying degrees of dynamism. Technical report, IMM Institute of Mathematical Modelling.

[96] Melachrinoudis, E., Ilhan, A. B., and Min, H. (2007). A dial-a-ride problem for client transportation in a health-care organization. Computers 86 Operations Research, $34(3): 742-759$.

[97] Mendoza, J. E., Castanier, B., Guéret, C., Medaglia, A. L., and Velasco, N. (2010). A memetic algorithm for the multi-compartment vehicle routing problem with stochastic demands. Computers \& Operations Research, 37(11):1886-1898. 
[98] Mendoza, J. E., Castanier, B., Guéret, C., Medaglia, A. L., and Velasco, N. (2011). Constructive heuristics for the multicompartment vehicle routing problem with stochastic demands. Transportation Science, 45(3):346-363.

[99] Mes, M., Van der Heijden, M., and Van Harten, A. (2007). Comparison of agent-based scheduling to look-ahead heuristics for real-time transportation problems. European Journal of Operational Research, 181(1):59-75.

[100] Mitrović-Minić, S., Krishnamurti, R., and Laporte, G. (2004). Double-horizon based heuristics for the dynamic pickup and delivery problem with time windows. Transportation Research Part B: Methodological, 38(8):669 - 685.

[101] Mitrović-Minić, S. and Laporte, G. (2004). Waiting strategies for the dynamic pickup and delivery problem with time windows. Transportation Research Part B: Methodological, 38(7):635-655.

[102] Montemanni, R., Gambardella, L. M., Rizzoli, A. E., and Donati, A. V. (2005). Ant colony system for a dynamic vehicle routing problem. Journal of Combinatorial Optimization, 10(4):327-343.

[103] Mu, Q., Fu, Z., Lysgaard, J., and Eglese, R. (2011). Disruption management of the vehicle routing problem with vehicle breakdown. Journal of the Operational Research Society, 62(4):742-749.

[104] Novoa, C. and Storer, R. (2009). An approximate dynamic programming approach for the vehicle routing problem with stochastic demands. European Journal of Operational Research, 196(2):509-515.

[105] Novoa, C. M. (2005). Static and dynamic approaches for solving the vehicle routing problem with stochastic demands. PhD thesis, Lehigh University, Pennsylvania, United States. AAT 3188502.

[106] Pankratz, G. and Krypczyk, V. (2009). Benchmark data sets for dynamic vehicle routing problems. http://www.fernuni-hagen.de/WINF/inhalte/benchmark_data. htm.

[107] Pillac, V., Guéret, C., and Medaglia, A. L. (2012). An event-driven optimization framework for dynamic vehicle routing. Decision Support Systems, Accepted manuscript.

[108] Potvin, J. Y., Xu, Y., and Benyahia, I. (2006). Vehicle routing and scheduling with dynamic travel times. Computers \&3 Operations Research, 33(4):1129-1137.

[109] Powell, W., Shapiro, J., and Simao, H. (2002). An adaptive dynamic programming algorithm for the heterogeneous resource allocation problem. Transportation Science, $36(2): 231-249$. 
[110] Powell, W. B. (2007). Approximate dynamic programming: solving the curses of dimensionality, volume 703 of Wiley Series in Probability and Statistics. WileyInterscience, Hoboken, New Jersey.

[111] Powell, W. B. (2009). What you should know about approximate dynamic programming. Naval Research Logistics, 56(3):239-249.

[112] Powell, W. B., Bouzaiene-Ayari, B., and Simao, H. (2007). Dynamic models for freight transportation. In Barnhart, C. and Laporte, G., editors, Transportation, volume 14 of Handbooks in Operations Research and Management Science, chapter 5, pages 285-365. North-Holland.

[113] Powell, W. B., Sheffi, Y., Nickerson, K. S., Butterbaugh, K., and Atherton, S. (1988). Maximizing profits for North American Van Lines' truckload division: A new framework for pricing and operation. Interfaces, 18(1):21-41.

[114] Powell, W. B. and Topaloglu, H. (2003). Stochastic programming in transportation and logistics. Handbooks in Operations Research and Management Science, 10:555-636.

[115] Powell, W. B. and Topaloglu, H. (2005). Fleet management. In Wallace, S. and Ziemba, W., editors, Applications of Stochastic Programming, volume 5 of MPS-SIAM series on Optimization, chapter 12, pages 185-215. SIAM.

[116] Psaraftis, H. (1980). A dynamic-programming solution to the single vehicle manyto-many immediate request dial-a-ride problem. Transportation Science, 14(2):130-154.

[117] Psaraftis, H. (1988). Dynamic vehicle routing problems. In Golden, B. and Assas, A., editors, Vehicle Routing: Methods and Studies, pages 223-248. Elsevier Science Publishers B.V.

[118] Psaraftis, H. N. (1995). Dynamic vehicle routing: Status and prospects. Annals of Operations Research, 61(1):143-164.

[119] Pureza, V. and Laporte, G. (2008). Waiting and buffering strategies for the dynamic pickup and delivery problem with time windows. INFOR, 46(3):165-175.

[120] Regan, A., Mahmassani, H., and Jaillet, P. (1995). Improving efficiency of commercial vehicle operations using real-time information: potential uses and assignment strategies. Transportation Research Record: Journal of the Transportation Research Board, 1493:188-198.

[121] Regan, A., Mahmassani, H., and Jaillet, P. (1998). Evaluation of dynamic fleet management systems - simulation framework. In Forecasting, Travel Behavior, And Network Modeling, number 1645 in Transportation Research Record, pages 176-184.

[122] Regan, A. C., Mahmassani, H. S., and Jaillet, P. (1996). Dynamic decision making for commercial fleet operations using real-time information. Transportation Research Record: Journal of the Transportation Research Board, 1537:91-97. 
[123] Rizzoli, A., Montemanni, R., Lucibello, E., and Gambardella, L. (2007). Ant colony optimization for real-world vehicle routing problems. Swarm Intelligence, 1:135-151.

[124] Romero, M., Sheremetov, L., and Soriano, A. (2007). A genetic algorithm for the pickup and delivery problem: An application to the helicopter offshore transportation. In Theoretical Advances and Applications of Fuzzy Logic and Soft Computing, volume 42 of Advances in Soft Computing, pages 435-444. Springer Berlin / Heidelberg.

[125] Roy, J. (2001). Recent trends in logistics and the need for real-time decision tools in the trucking industry. In System Sciences, 2001. Proceedings of the 34th Annual Hawaii International Conference on.

[126] Secomandi, N. (2000). Comparing neuro-dynamic programming algorithms for the vehicle routing problem with stochastic demands. Computers $\& 3$ Operations Research, 27(11-12):1201 - 1225 .

[127] Secomandi, N. (2001). A rollout policy for the vehicle routing problem with stochastic demands. Operations Research, 49(5):796-802.

[128] Secomandi, N. and Margot, F. (2009). Reoptimization approaches for the vehiclerouting problem with stochastic demands. Operations Research, 57(1):214-230.

[129] Simao, H., Day, J., George, A., Gifford, T., Nienow, J., and Powell, W. B. (2009). An approximate dynamic programming algorithm for large-scale fleet management: A case application. Transportation Science, 43(2):178-197.

[130] Slater, A. (2002). Specification for a dynamic vehicle routing and scheduling system. International Journal of Transport Management, 1(1):29 - 40.

[131] Sleator, D. and Tarjan, R. (1985). Amortized efficiency of list update and paging rules. Communications of the ACM, 28(2):202-208.

[132] Smolic-Rocak, N., Bogdan, S., Kovacic, Z., and Petrovic, T. (2010). Time windows based dynamic routing in multi-agv systems. IEEE Transactions on Automation Science and Engineering, 7(1):151-155.

[133] Solomon, M. M. (1987). Algorithms for the vehicle-routing and scheduling problems with time window constraints. Operations Research, 35(2):254-265.

[134] Spivey, M. and Powell, W. B. (2004). The dynamic assignment problem. Transportation Science, 38(4):399-419.

[135] Stahlbock, R. and Voss, S. (2008). Operations research at container terminals: a literature update. OR Spectrum, 30(1):1-52.

[136] Tagmouti, M., Gendreau, M., and Potvin, J.-Y. (2011). A dynamic capacitated arc routing problem with time-dependent service costs. Transportation Research Part C: Emerging Technologies, 19(1):20 - 28. 
[137] Taillard, E., Badeau, P., Gendreau, M., Guertin, F., and Potvin, J. (1997). A tabu search heuristic for the vehicle routing problem with soft time windows. Transportation Science, 31(2):170-186.

[138] Taillard, E. D., Gambardella, L. M., Gendreau, M., and Potvin, J.-Y. (2001). Adaptive memory programming: A unified view of metaheuristics. European Journal of Operational Research, 135(1):1 - 16.

[139] Taniguchi, E. and Shimamoto, H. (2004). Intelligent transportation system based dynamic vehicle routing and scheduling with variable travel times. Transportation Research Part C: Emerging Technologies, 12(3-4):235-250.

[140] Taniguchi, E. and Thompson, R. (2002). Modeling city logistics. Transportation Research Record: Journal of the Transportation Research Board, 1790(1):45-51.

[141] Thomas, B. W. (2007). Waiting strategies for anticipating service requests from known customer locations. Transportation Science, 41(3):319-331.

[142] Thomas, B. W. and White, Chelsea C., I. (2004). Anticipatory route selection. Transportation Science, 38(4):473-487.

[143] Toth, P. and Vigo, D., editors (2002). The vehicle routing problem, volume 9 of SIAM Monographs on Discrete Mathematics. SIAM Philadelphia.

[144] Van Hemert, J. I. and Poutré, J. L. (2004). Dynamic routing problems with fruitful regions: Models and evolutionary computation. In Yao, X., Burke, E., Lozano, J. A., Smith, J., Merelo-Guervós, J. J., Bullinaria, J. A., Rowe, J., Tino, P., Kabán, A., and Schwefel, H.-P., editors, Parallel Problem Solving from Nature, volume 3242 of Lecture Notes in Computer Science, pages 692-701. Springer Berlin / Heidelberg.

[145] Van Hentenryck, P. and Bent, R. (2006). Online stochastic combinatorial optimization. MIT Press.

[146] Verweij, B., Ahmed, S., Kleywegt, A., Nemhauser, G., and Shapiro, A. (2003). The sample average approximation method applied to stochastic routing problems: a computational study. Computational Optimization and Applications, 24(2):289-333.

[147] Waters, C. (1989). Vehicle-scheduling problems with uncertainty and omitted customer. The Journal of the Operational Research Society, 40(12riz):1099-1108.

[148] Wilson, N. and Colvin, N. (1977). Computer control of the Rochester dial-a-ride system. Technical Report Report R77-31, Dept. of Civil Engineering, Massachusetts Institute of Technology, Cambridge, Massachusetts.

[149] Xiang, Z., Chu, C., and Chen, H. (2008). The study of a dynamic dial-a-ride problem under time-dependent and stochastic environments. European Journal of Operational Research, 185(2):534-551. 
[150] Yang, J., Jaillet, P., and Mahmassani, H. (2004). Real-time multivehicle truckload pickup and delivery problems. Transportation Science, 38(2):135-148.

[151] Yang, S., Hamedi, M., and Haghani, A. (2005). Online dispatching and routing model for emergency vehicles with area coverage constraints. In Network Modeling 2005, number 1923 in Transportation Research Record, pages 1-8.

[152] Yao, Y., Ergun, O., and Johnson, E. (2007). Integrated model for the dynamic on-demand air transportation operations. In Zeimpekis, V., Tarantilis, C. D., Giaglis, G. M., and Minis, I., editors, Dynamic Fleet Management, volume 38 of Operations Research/Computer Science Interfaces Series, pages 95-111. Springer US. 10.1007/9780-387-71722-7_5.

[153] Zeimpekis, V., Minis, I., Mamassis, K., and Giaglis, G. M. (2007a). Dynamic management of a delayed delivery vehicle in a city logistics environment. In Zeimpekis, V., Tarantilis, C. D., Giaglis, G. M., and Minis, I., editors, Dynamic Fleet Management, volume 38 of Operations Research/Computer Science Interfaces Series, chapter 9, pages 197-217. Springer US.

[154] Zeimpekis, V., Tarantilis, C. D., Giaglis, G. M., and Minis, I., editors (2007b). Dynamic Fleet Management, volume 38 of Operations Research Computer Science Interfaces Series. Springer US. 